Review

\title{
The Nervous System Cytoskeleton under Oxidative Stress
}

\section{John Gardiner *, Robyn Overall and Jan Marc}

School of Biological Sciences, The University of Sydney, Science Road, Macleay Building A12, Camperdown 2006, Australia; E-Mails: robyn.overall@sydney.edu.au (R.O.); jan.marc@sydney.edu.au (J.M.)

* Author to whom correspondence should be addressed; E-Mail: jgardiner@mail.usyd.edu.au; Tel.: +612-9351-2383; Fax: +612-9351-4771.

Received: 10 September 2013; in revised form: 30 September 2013/Accepted: 12 October 2013 / Published: 21 October 2013

\begin{abstract}
Oxidative stress is a key mechanism causing protein aggregation, cell death and neurodegeneration in the nervous system. The neuronal cytoskeleton, that is, microtubules, actin filaments and neurofilaments, plays a key role in defending the nervous system against oxidative stress-induced damage and is also a target for this damage itself. Microtubules appear particularly susceptible to damage, with oxidative stress downregulating key microtubule-associated proteins [MAPs] and affecting tubulin through aberrant post-translational modifications. Actin filaments utilise oxidative stress for their reorganisation and thus may be less susceptible to deleterious effects. However, because cytoskeletal components are interconnected through crosslinking proteins, damage to one component affects the entire cytoskeletal network. Neurofilaments are phosphorylated under oxidative stress, leading to the formation of protein aggregates reminiscent of those seen in neurodegenerative diseases. Drugs that target the cytoskeleton may thus be of great use in treating various neurodegenerative diseases caused by oxidative stress.
\end{abstract}

Keywords: microtubule; actin; neurofilament; posttranslational modification

\section{Abbreviations}

Abl: Abelson murine leukaemia viral oncogene homolog 1; ADF: actin depolymerising factor; ADNP: activity-dependent neuroprotective protein; APP: amyloid precursor protein; Arg: Abl-related gene; BPAG: bullous pemphigoid antigen; Cdk5: cell division kinase 5; CRMP: collapsin response-mediator 
proteins; DAT: dopamine transporter; EB1: end binding 1; GSH: glutathione; GSK: glycogen synthase kinase; HDAC6: histone deacetylase 6; HNE: 4-hydoxy-2-nonenal; LKE: lanthionine ketimine; L-NAME: $\mathrm{N}$ omega-nitro-L-arginine methyl ester; MAP: microtubule-associated protein; NADPH: nicotinamide adenine dinucleotide phosphate; NAP: NAPVSIPQ; NF: neurofilament; NMDA: N-methyl-D-aspartic acid; PCOOHs: phosphatidylcholine hydroperoxides; Shot: short stop; UCH-L1: ubiquitin $C$-terminal hydrolase L1.

\section{Introduction}

Oxidative stress represents an imbalance between the production of reactive oxygen species and the ability of the biological system to detoxify the reactive intermediates or to repair the resultant damage. Oxidative stress leads to the formation of peroxides and free radicals, which damage proteins, lipids and DNA. Oxidative stress is implicated in various neurodegenerative disorders, largely due to the abnormal accumulation of oxidatively damaged macromolecules, including $\beta$-amyloid and Tau [1].

The neuronal cytoskeleton plays a key role in both protecting cells against oxidative stress and is itself the target of oxidative stress-induced damage. The neuronal cytoskeleton consists of microtubules, actin filaments and neurofilaments (intermediate filament proteins). All three are linked through crosslinking proteins to form a unified whole. These cytoskeletal components are regulated through changes in expression levels, post-translational modifications and the effects of the binding of partner proteins. Oxidative stress affects the regulation of the neuronal cytoskeleton through these regulatory mechanisms, with some changes being protective and others pathogenic. Thus, the cytoskeleton plays a key role in cellular responses to oxidative stress and the onset of oxidative stress-induced neurodegeneration.

\section{Microtubules and Oxidative Stress in the Nervous System}

\subsection{Drug Studies Indicate a Role for Microtubules in Oxidative Stress}

The stabilisation of microtubules with taxol or destabilisation with vinblastine causes concentration-dependent cell death with apoptotic features in neurons. Treatment with taxol increases the amount of reactive oxygen species, and inhibition of nicotinamide adenine dinucleotide phosphate (NADPH) oxidase or suppression of gp91 (phox) attenuates taxol-induced neuronal death, although the use of NADPH oxidase inhibitors does not decrease vinblastine-induced cell death [2]. The microtubule depolymerising agent, colchicine, upregulates genes related to oxidative stress, glutathione peroxidise 1 and catalase, in rat cerebellar granule neurons [3]. Colchicine causes loss of cholinergic neurons and cognitive dysfunction that is associated with excessive production of free radicals, including malondialdehyde and nitrite, and a decrease in reduced glutathione [4]. Treatment of primary mesencephalic neurons with microtubule-depolymerising agent stimulates dopamine transporter (DAT) activity, leading to an increased rate of dopamine uptake and increased levels of dopamine-induced oxidative stress along with accelerated cell death. DAT co-immunoprecipitates with $\alpha$ - and $\beta$-tubulin, suggesting a functional link between the microtubule cytoskeleton and DAT [5]. 


\subsection{The Proteasome, Oxidative Stress and Microtubules}

Oxidative stress causes the aggregation of a number of proteins implicated in neurodegenerative disorders [1]. Mutations that affect the dynein motor machinery are sufficient to cause motor neuron disease. Indeed, decreased dynein function impairs autophagic clearance of aggregate-prone proteins [6], losing its function in aggresome formation and failing to return autophagosomes and lysosomes to the centre of the cell for degradation [7]. An intact microtubule cytoskeleton is essential for aggresome formation, and cytoplasmic dynein/dynactin is responsible for the directed transport of misfolded protein into aggresomes [8]. Thus, breakdown of the microtubule cytoskeleton under oxidative stress will, in turn, increase the load of aggregated protein in a cell, potentially leading to overload and cell death.

\section{Role of Post-Translational Modifications of Tubulin}

\subsection{Acetylation}

HDAC6 is responsible for the deacetylation of $\alpha$-tubulin in the brain. An inhibitor of HDAC6, tubastatin A, induces elevated levels of $\alpha$-tubulin, but not histone, in primary cortical neuron cultures. Tubastatin A also confers dose-dependent protection of neurons against glutathione depletion-induced oxidative stress [9]. Microtubule acetylation is a post-translational modification that promotes the binding and transport of kinesin-1 in neurons [10]. Multiple organelles are transported along microtubules by kinesins, and the breakdown of this process can lead to increased oxidative stress in neurons.

Oxidative stress caused by hydrogen peroxide leads to axonal swelling and beading, consistent with disruption of microtubules by oxidative stress and subsequent hold-up of axonal transport [11]. HDAC6 plays an important role in the modulation of mitochondrial transport. When inhibited with the specific HDAC6 inhibitor, tubacin, more kinesin-1 associates with mitochondria. HDAC6 activity is regulated by GSK3 $\beta$ via phosphorylation [12]. Mitochondria in Alzheimer's disease brains are perinuclear, with few organelles in distal processes, where they are normally located in healthy cells and are needed for exocytosis, ion channel pumps, synaptic function and other activities. Alzheimer's disease neurons thus have an increased amount of reactive oxidative species and decreased metabolic capability [13]. Axonal transport of mitochondria and mitochondrial dynamics may also be disrupted in amyotrophic lateral sclerosis [14] and in retinal ganglion cell neurodegeneration [15], where oxidative stress is again a factor. Indeed, mitochondrial dysfunction is thought to play a key role in disease pathogenesis, because neurons are among the most energy-consuming cell types and have a highly developed cell shape [16].

Tau protein inhibits kinesin-dependent transport of peroxisomes into neurites, and loss of peroxisomes makes cells vulnerable to oxidative stress [17]. Here, again, tubulin acetylation may play a key role in promoting peroxisomal transport to distal processes, and the oxidative stress-induced breakdown of microtubules may lead to increased oxidative stress in these processes. Finally, Parkinson's disease toxins, such as rotenone, disrupt vesicle transport along microtubules, leading to increased accumulation of vesicles in the cell body and increased cytosolic concentration of dopamine, due to leakage from the vesicles. Elevated oxidative stress induced by dopamine oxidation may trigger apoptosis of dopaminergic neurons [18]. 
Loss of $\alpha$-tubulin acetylation and impaired motor-protein transport are implicated in a number of neurological disorders, including Huntington's disease [19], familial dysautonomia [20] and amyotrophic lateral sclerosis, all of which occur through disruption of the $\alpha$-tubulin acetylase activity of the Elongator complex [21]. These diseases are also characterised by oxidative stress [22]. It seems likely that the breakdown in transport of cargoes along microtubules due to defects in tubulin acetylation may play a key role in promoting oxidative stress and subsequent neurodegeneration, whereas increased $\alpha$-tubulin acetylation and microtubule-based transport may act to alleviate oxidative stress. Transcription factor trafficking is also affected in oxidatively-stressed neurons [23].

\subsection{3-Nitrotyrosination}

The conjugated aromatic ring of tyrosine is a target for free-radical attack, leading to the formation of 3-nitrotyrosine under conditions of oxidative stress [24]. $\alpha$-Tubulin is tyrosinated at its $C$-terminus by the action of a tubulin-tyrosine ligase, and the $C$-terminal tyrosine can then be removed by a tubulin carboxypeptidase. This post-translational modification prevents the binding of kinesin-1 to microtubules in dendrites, thus navigating it to axonal microtubules [25]. High glucose and hyperglycemia can result in the production of high concentrations of reactive oxygen species with subsequent damage to the central nervous system. Indeed, in neuroblastoma and glioma cells exposed to D-glucose, there is a downregulation of tubulin isoforms and an increase in 3-nitrotyrosine modification of tubulin [26]. There is some controversy over the effect of 3-nitrotyrosine incorporation into tubulin on microtubule function. One study found that 3-nitrotyrosine incorporation into mammalian cells by tubulin-tyrosine ligase was irreversible and caused changes in microtubule organisation, cell morphology and the redistribution of the motor protein, dynein [27]. However, another study found that the incorporation of 3-nitrotyrosine into microtubules was reversible and not detrimental to dividing cells [28]. Therefore, the effect of 3-nitrotyrosine incorporation into microtubules is still an open question, but it may interfere with the correct localisation of neuronal motor proteins and MAPs.

\subsection{Glutathionylation}

Friedreich's ataxia is caused by mutations in the gene for frataxin, a mitochondrial protein implicated in iron metabolism. Loss of frataxin causes iron overload in tissues and an increase in free-radical production and oxidation. There is a significant rise in the dynamic pool of tubulin, possibly caused by increased protein glutathionylation [29]. Loss of glutathionylation of cytoskeletal proteins may give rise to oxidative stress, as this is a way that cells regulate microtubule cytoskeletal stability [29]. It has been recently suggested that microtubule glutathionylation, an increased intracellular level of oxidized glutathione, may cause an alteration of the cytoskeleton organisation and function, leading to axon degeneration [30].

\section{Peroxidation of Lipids Damages Microtubules}

Lipid peroxidation is regarded as a major cause of brain damage by oxygen radicals. The antioxidant, melatonin, prevents lipid peroxidation caused by okadaic acid in neuroblastoma cells and 
prevents the disruption of the microtubule cytoskeleton [31]. Phosphatidylcholine hydroperoxides (PCOOHs) disrupt both the formation of neurites and neuronal microtubules. Differentiated cells are less susceptible to this form of attack than undifferentiated cells [32]. Another product of lipid peroxidation, acrolein, disrupts microtubules in PC12 cells, as well as sympathetic ganglion cells in vitro [33], and this may be due in part to increased phosphorylation of Tau protein [34]. In a model system of microtubule degradation by lipid-derived free radicals, it was shown that the kinetics of this process is determined by the level of lipid saturation and the presence of free radical scavengers [35]. Oxidised very-low-density lipoprotein and hydrogen peroxide reduced phosphorylation of the microtubule-destabilising protein, stathmin, whereas the lipid peroxidation product, 4-hydroxy-2-nonenal (HNE; an aldehyde), increased it, suggesting that free radicals, but not aldehydes, may play a key role in neuronal cell death caused by lipoprotein oxidation [36]. However, HNE disrupts microtubules and modifies cellular tubulin via Michael addition [37], as well as inhibiting dephosphorylation of Tau protein [38].

\section{Oxidative Stress and MAPs}

Oxidative stress leads to changes in the suite of MAPs found in neurons through changes in expression levels, post-translational modification, oxidative damage and breakdown of proteins by proteolysis.

\subsection{Changes in MAP Expression Levels under Oxidative Stress}

Expression levels of various MAPs change under conditions of oxidative stress in neurons. Some of these MAPs-MAP2, Tau and doublecortin, are microtubule-stabilising proteins, and the reduction in their expression levels may serve to destabilise microtubules. A proteomic analysis of proteins differentially regulated under acute oxidative stress showed that a number of MAPs changed their levels of expression. Doublecortin was downregulated. Collapsin response-mediator proteins (CRMPs) were upregulated. $\alpha$-tubulin 6 was downregulated, and Tau protein and MAP2 were downregulated [39]. Other studies support these findings. Chronic ozone exposure causes an increase of reactive oxygen species and oxidative stress. Ozone causes cell swelling in neurons and a decrease in the MAP doublecortin and, also, chronic loss of brain repair in the hippocampus in adult rats [40].

Lanthionine ketimine (LK) is a member of a class of thioethers that are present in the mammalian central nervous system. LK interacts with CRMP2 and decreases CRMP2 coprecipitation with $\beta$-tubulin. A cell-permeable LK-ester, LKE, protects against oxidative challenge with hydrogen peroxide [41]. CRMP2 is a GTPase-activating protein, and this activity is important for microtubule assembly and neurite formation [42]. However, the fact that CRMPs are upregulated under oxidative stress and tubulin-associated CRMP decreases following treatment of neurons with neuroprotective LK indicates that CRMP has a role in promoting oxidative stress alterations of the microtubule cytoskeleton.

\subsection{Oxidative Stress Modifies MAPs and Tubulin}

Soluble oligomeric forms of amyloid beta-peptide induce oxidative stress in neurons, and this oxidative stress leads to the proteolysis of MAP1A, MAP1B and MAP2 by caspase-3 and calpain [43]. Quinones modify tubulin, leading to its aggregation into dimers and other oligomers [44], which 
disrupt microtubule formation. The I93M mutation in ubiquitin $C$-terminal hydrolase L1 (UCH-L1) is associated with familial Parkinson's disease. UCH-L1 is a major target of oxidative/carbonyl damage associated with sporadic Parkinson's disease. Familial Parkinson's disease-associated and carbonyl-modified UCH-L1 in sporadic Parkinson's disease share aberrant properties, with both modified forms modulating tubulin polymerisation, and this gain of function may be relevant to both familial and sporadic Parkinson's disease [45]. M sulfoxides map to neuron-enriched $\beta$-tubulin in Alzheimer's disease brains, suggesting oxidative modification of $\beta$-III tubulin as a contributor to the neuronal cytoskeletal disruption characteristic of Alzheimer's disease [46].

Tau protein is implicated in neurodegenerative diseases, including Alzheimer's disease. Peroxynitrite induces the oxidative crosslinking and site-selective nitration of Tau monomers. This causes the formation of aggregates that are crosslinked by inter-filament bridges, inhibiting the ability of monomeric Tau to promote tubulin assembly [47]. Arg, a member of the Abl family of tyrosine kinases, phosphorylates Tau protein at Y394. Arg plays a key role in both oxidative stress response and neural development. Paired helical filaments that comprise neurofibrillary tangles in Alzheimer's disease contain an abnormally hyperphosphorylated form of Tau [48]. During mitochondrial inhibition and oxidative stress, actin-depolymerising factor (ADF)/cofilin assemble into rods along the processes of cultured neurons and recruit phosphorylated Tau protein. Microtubules are disrupted in processes that possess rods [49], suggesting that neurofibrillary tangles may be initiated by F-actin bundling caused by oxidative stress [50]. Oxidised products of dopamine (dopamine quinone) also promote Tau polymerisation, again suggesting a link between oxidative damage and the onset of tauopathies [51]. The peptidyl-prolyl cis/trans isomerase Pin 1 regulates the function and processing of both Tau proteins and amyloid precursor protein (APP), both of which are implicated in Alzheimer's disease. In humans, the Alzheimer's disease brain Pin1 is downregulated by oxidative modification and/or genetic changes [52].

\subsection{MAPs May Act to Protect Neurons against Oxidative Stress}

Some MAPs appear to function to decrease oxidative stress in neurons. NAPVSIPQ (NAP), an 8 -amino acid peptide derived from activity-dependent neuroprotective protein (ADNP), interacts with microtubules and protects microtubules from degradation under oxidative stress, probably inhibiting an early event of apoptosis [53]. NAP specifically binds BIII-tubulin, and taxol reduces NAP-tubulin binding [54]. Parkin is a MAP that is mutated in familial Parkinson's disease. Elevating Parkin in cells reduces markers of oxidative stress, whereas blocking its expression increases oxidative stress. Parkin also acts as a ubiquitin ligase in the ubiquitin-proteasome system, and mitochondrial function is deficient in mouse and fly Parkin knockdown models. It is unclear which of Parkin's functions is neuroprotective [55]. Hydrogen peroxide treatment of neuronal and non-neuronal mixed rat retinal cell cultures causes the loss of MAP2 in the neuronal soma, and downregulation of MAP2 increases the vulnerability of retinal neurons to oxidative stress [56].

\section{Tensegrity: Interactions between Microtubules, Actin and Neurofilaments in the Nervous System}

Tensional integrity (tensegrity) architecture has been proposed to be a means by which cells stabilise their shape and sense mechanical signals from the nanoscale to the macroscale [57]. 
Importantly, these networks are in a state of isometric tension (i.e., they experience a tensile prestress), ensuring that molecular-scale mechanochemical transduction mechanisms proceed simultaneously [58]. In cells, it is suggested that microtubules provide compression-resistant components and actin-filaments, tensional elements, in a unified tensegrity architecture whole. There is evidence to support this theory, with living cells behaving like discrete structures composed of an interconnected network of actin filaments and microtubules when mechanical stresses are applied to cell surface integrin receptors [59].

Short Stop (Shot) encodes a neuronally expressed family of proteins required for sensory and motor axon extension in Drosophila melanogaster. Shot isoforms contain an $N$-terminal F-actin and $C$-terminal microtubule-binding domains that crosslink F-actin and microtubules in cultured cells. Both the F-actin and microtubule-binding domains are required in the same molecule for axon extension [60]. Shots also play a key role in dendrite formation and axonal terminal arborisation at the neuromuscular junction, forming a complex with the microtubule plus-end-binding (+TIP) protein, EB1 [61]. Thus, there is evidence for the necessity of a linkage between microtubules and actin filaments in neuronal development, possibly due to the reliance of neurons upon cytoskeletal tensegrity architecture to maintain prestress.

Actin and neurofilaments are also crosslinked by dystonin, a member of the bullous pemphigoid antigen (BPAG) family of plakins [62]. Disruption of dystonin causes sensory neuropathy in mice and disorganisation of neurofilaments and microtubules [63]. Neurofilaments undergo strain stiffening suggesting that, as well as microtubules, they act as compression-resistant components of neuronal cytoskeletal tensegrity architecture [64]. Any perturbation of actin filaments, microtubules or neurofilaments, for example, by oxidative stress, will lead to a loss of prestress and the ability of the cytoskeleton to function as an integrated whole.

\section{Actin and Oxidative Stress in the Nervous System}

\subsection{Actin and Actin-Binding Proteins Respond to Oxidative Stress}

Interestingly, although actin and actin-binding proteins respond to oxidative stress, they appear less susceptible than microtubules. This may be in part due to the fact that the actin cytoskeleton actually utilises oxidative stress as a developmental mechanism.

Oxidative stress increases cellular actin aggregation and the binding of PINK1 to Parkin, both of which proteins are implicated in Parkinson's disease. Parkin also associates with actin, suggesting that oxidative stress may disrupt actin organisation through changing the binding substrates of Parkin [65]. $\mathrm{Cu}^{2+}$-induced stress causes glutathione (GSH) adducts of cysteines of synaptosomal actin followed by its deglutathionylation and dimerisation. Torpedo cyclophilin B is able to sustain peroxiredoxin-1 activity and, thus, might be involved in presynaptic defence against oxidative stress affecting G-actin [66]. Oxidative stress results in a rapid dephosphorylation of cellular cofilin (an actin-binding protein) prior to its assembly into rod-shaped inclusions. The ATP-sensitive interaction of cofilin phosphatase chronophin with chaperone hsp90 mediates the formation of cofilin/actin rods [67].

TNF alpha and II-1beta stimulate a redox-dependent reorganisation of the actin cytoskeleton into lamellipodia under the regulation of Rac1 and a neuronal NADPH oxidase as the source of reactive 
oxygen species. However, the persistent presence of reactive oxygen species provoked carbonylation of actin, which coincided with the loss of lamellipodia and the arrest of cellular plasticity [68]. Carbonylation of $\beta$-actin was also detected following the production of reactive oxygen species following ischemia-reperfusion in the hippocampus of the macaque monkey [69].

\subsection{Glial and Astrocyte Actin Cytoskeletons}

Astrocytes and glia modify their actin cytoskeletons to protect neurons against oxidative stress. Homocysteine is an excitatory amino acid that enhances the vulnerability of neuronal cells to oxidative injury. In astrocytes exposed to homocysteine, there is a dramatic change in the actin cytoskeleton and a change in cell morphology to fusiform and/or flattened cells with retracted cytoplasm. Neurons do not appear to change their actin cytoskeletal organisation in response to homocysteine. Thus, astrocytes reorganise their actin cytoskeleton in response to oxidative stress in order to protect neurons from its effects [70]. The oxidant, paraquat, induces rapid differentiation and proliferation of glial cells, including the expression of smooth muscle actin. In pure neuronal cultures, paraquat caused apoptosis of photoreceptors and amacrine neurons, and coculturing neurons with glial cells completely prevented this apoptosis. Thus, neuronal oxidative stress is ameliorated by the reorganisation of the actin cytoskeleton in glial cells [71].

\section{Neurofilaments and Oxidative Stress}

\subsection{Neurofilament Phosphorylation and Oxidative Stress}

Aggregation of neurofilament (NF) protein because of phosphorylation is a key process in neurodegenerative diseases, including Charcot-Marie-Tooth disease [72], Alzheimer's disease and amyotrophic lateral sclerosis [73]. Injection of quinolinic acid increases phosphorylation of the low molecular weight NF subunit in neurons, whereas N-methyl-D-aspartic acid (NMDA) antagonist MK-801 and the antioxidant, $\mathrm{N}$ omega-nitro-L-arginine methyl ester (L-NAME), prevent this hyperphosphorylation. The hyperphosphorylation is targeted at the Ser55 phosphorylating site on the NFL head domain, a regulatory site for NF assembly in vivo [74]. In the spinal cord in Friedreich's ataxia, there is an abnormal distribution of phosphorylated forms of NFs [75]. Pin1 modulates oxidative stress-induced phosphorylation of high molecular weight NF protein. Cyclin-dependent kinase 5 (cdk5) phosphorylates NF protein, and overexpression of cdk5 inhibits NF axonal transport and induces accumulation of disordered phosphor-NF cables. Oxidative stress recapitulates this phenotype in a cdk5-dependent manner [76].

\subsection{Oxidative Stress Modifies Neurofilaments}

The lipid peroxidation product, acrolein, leads to the generation of protein carbonyl compounds and the formation of NF aggregates by dityrosine crosslinking. These aggregates display thioflavin $\mathrm{T}$ reactivity, reminiscent of amyloid [77]. In Alzheimer's disease brains, there is an increase in $\mathrm{N}$-malondialdehyde-lysine formation targeting NFL proteins, suggesting an important role for lipid-peroxidation-derived protein modification in Alzheimer's disease pathogenesis [78]. Neuroaxonal spheroid aggregates in leukoencephalopathy contain amyloid and NF proteins, and there 
is an increase in markers for oxidative stress [79]. The lipid peroxidation product, HNE, causes the formation of adducts in NF proteins, but here, it is suggested that HNE may play a protective role against toxic aldehydes resulting from oxidative damage, due to its consistency from birth to senility and its dependence on phosphorylation [80]. Modification of NFs by oxidative advanced glycation end-products in diabetic neuropathy leads to axonal degeneration [81]. Oxidative stress also affects the neuronal cytoskeleton through proteolysis and/or abnormal structural changes in NFs [82], consistent with antioxidant treatment of dogs, which leads to a decrease in protein carbonyl levels of NF triplet L protein [83].

\section{Conclusions}

We have demonstrated that the nervous system cytoskeleton plays a key role in oxidative stress responses and the onset of oxidative stress-induced neurodegeneration. Drugs that target the cytoskeleton, for example, the HDAC6 inhibitor, tubastatin, may thus be of great use in treating various neurodegenerative diseases caused by oxidative stress. Diet, too, may be important, with zinc availability, for example, causing alterations in tubulin thiol redox status [84]. The development of a polypeptide inhibitor of cdk5, potentially useful in reducing protein aggregation caused by the phosphorylation of NF and Tau proteins, is undergoing tests in mice [85]. The development of an inhibitor against the Elongator complex, $\alpha$-tubulin acetylase, would provide key insights into the role of tubulin acetylation in oxidative stress responses, while a proteomic approach to the identification of cytoskeleton-associated proteins under oxidative stress conditions would be of great interest.

\section{Conflicts of Interest}

The authors declare no conflict of interest.

\section{References}

1. Grimm, S.; Hoehn, A.; Davies, K.J.; Grune, T. Protein oxidative modifications in the ageing brain, consequence for the onset of neurodegenerative disease. Free Radic. Res. 2010, 45, 73-88.

2. Jang, H.J.; Hwang, S.; Cho, K.Y.; Kim do, K.; Chay, K.O.; Kim, J.K. Taxol induces oxidative neuronal cell death by enhancing the activity of NADPH oxidase in mouse cortical cultures. Neurosci. Lett. 2008, 443, 17-22.

3. Yeste-Velasco, M.; Alvira, D.; Sureda, F.X.; Rimbau, V.; Forsby, A.; Pallàs, M.; Camins, A.; Folch, J. DNA low-density array analysis of colchicines neurotoxicity in rat cerebellar granular neurons. Neurotoxicology 2008, 29, 309-317.

4. Kumar, A.; Naidu, P.S.; Seghal, N.; Padi, S.S. Neuroprotective effects of resveratrol against intracerebroventricular colchicines-induced cognitive impairment and oxidative stress in rats. Pharmacology 2007, 79, 17-26.

5. Wersinger, C.; Sidhu, A. Disruption of the interaction of alpha-synuclein with microtubules enhances cell surface recruitment of the dopamine transporter. Biochemistry 2005, 44, 13612-13624. 
6. Ravikumar, B.; Acevedo-Arozena, A.; Imarisio, S.; Berger, Z.; Vacher, C.; O'Kane, C.J.; Brown, S.D.; Rubinsztein, D.C. Dynein mutations impair autophagic clearance of aggregate-prone proteins. Nat. Genet. 2005, 37, 771-776.

7. Cai, Z.L.; Shi, J.J.; Yang, Y.P.; Cao, B.Y.; Wang, F.; Huang, J.Z.; Yang, F.; Zhang, P.; Liu C,F. $\mathrm{MPP}^{+}$impairs autophagic clearance of alpha-synuclein by impairing the activity of dynein. Neuroreport 2009, 20, 569-573.

8. Johnston, J.A.; Illing, M.E.; Kopito, R.R. Cytoplasmic dyneindynactin mediates the assembly of aggresomes. Cell Motil. Cytoskel. 2002, 53, 26-38.

9. Butler, K.V.; Kalin, J.; Brochier, C.; Vistoli, G.; Langley, B.; Kozikowski, A.P. Rational design and simple chemistry yield a superior, neuroprotective HDAC6 inhibitor, tubastatin A. J. Am. Chem. Soc. 2010, 132, 10842-10846.

10. Reed, N.A.; Cai, D.; Blasius, T.L.; Jih, G.T.; Meyhofer, E.; Gaertig, J.; Verhey, K.J. Microtubule acetylation promotes kinesin-1 binding and transport. Curr. Biol. 2006, 16, 2166-2172.

11. Roediger, B.; Armati, P.J. Oxidative stress induces axonal beading in cultured human brain tissue. Neurobiol. Dis. 2003, 13, 222-229.

12. Chen, S.; Owens, G.C.; Makarenkova, H.; Edelman, D.B. HDAC6 regulates mitochondrial transport in hippocampal neurons. PLoS One 2010, 5, e10848.

13. Bonda, D.J.; Wang, X.; Perry, G.; Smith, M.A.; Zhu, X. Mitochondrial dynamics in Alzheimer's disease, opportunities for future treatment strategies. Drugs Aging 2010, 27, 181-192.

14. Shi, P.; Gal, J.; Kwinter, D.M.; Liu, X.; Zhu, H. Mitochondrial dysfunction in amyotrophic lateral sclerosis. Biochim. Biophys. Acta 2010, 1802, 45-51.

15. Carelli, V.; La Morgia, C.; Valentino, M.L.; Barboni, P.; Ross-Cisneros, F.N.; Sadun, A.A. Retinal ganglion cell neurodegeneration in mitochondrial inherited disorders. Biochim. Biophys. Acta 2009, 1787, 518-528.

16. Itoh, K.; Nakamura, K.; Iijima, M.; Sesaki, H. Mitochondrial dynamics in neurodegeneration. Trends Cell Biol. 2013, 23, 64-71.

17. Stamer, K.; Vogel, R.; Thies, E.; Mandelkow, E.; Mandelkow, E.M. Tau blocks traffic of organelles, neurofilaments, and APP vesicles in neurons and enhances oxidative stress. J. Cell Biol. 2002, 16, 1051-1063.

18. Feng, J. Microtubule, a common target for parkin and Parkinson's disease toxins. Neuroscientist 2006, 12, 469-476.

19. Dompierre, J.P.; Godin, J.D.; Charrin, B.C.; Cordeliéres, F.P.; King, S.J.; Humbert, S.; Saudou, F. Histone deacetylase 6 inhibition compensates for the transport deficit in Huntington's disease by increasing tubulin acetylation. J. Neurosci. 2007, 27, 3571-3583.

20. Gardiner, J.; Barton, D.; Marc, J.; Overall, R. Potential role of tubulin acetylation and microtubule-based protein trafficking in familial dysautonomia. Traffic 2007, 8, 1145-1149.

21. Nguyen, L.; Humbert, S.; Saudou, F.; Chariot, A. Elongator-An emerging role in neurological disorders. Trends Mol. Med. 2010, 16, 1-6.

22. Gardiner, J.; Barton, D.; Overall, R.; Marc, J. Neurotrophic support and oxidative stress, converging effects in the normal and diseased nervous system. Neuroscientist 2009, 15, 47-61.

23. Patel, V.P.; Defranco, D.B.; Chu, C.T. Altered transcription factor trafficking in oxidatively-stressed neuronal cells. Biochim. Biophys. Acta 2012, 1822, 1773-1782. 
24. Jomova, K.; Vondrakova, D.; Lawson, M.; Valko, M. Metals, oxidative stress and neurodegenerative disorders. Mol. Cell. Biochem. 2010, 345, 91-104.

25. Konishi, Y.; Setou, M. Tubulin tyrosination navigates the kinesin-1 motor domain to axons. Nat. Neurosci. 2009, 12, 559-567.

26. Gadau, S.; Lepore, G.; Zedda, M.; Mura, A.; Farina, V. Different nitroative-induced microtubular modifications and testosterone neuroprotective effects on high-D-glucose-exposed neuroblastoma and glioma cells. Neuro. Endocrinol. Lett. 2009, 30, 515-524.

27. Eiserich, J.P.; Estévez, A.G.; Bamberg, T.V.; Ye, Y.Z.; Chumley, P.H.; Beckman, J.S.; Freeman, B.A. Microtubule dysfunction by posttranslational nitrotyrosination of alpha-tubulin, a nitric oxide-dependent mechanism of cellular injury. Proc. Natl. Acad. Sci. USA 1999, 96, 6365-6370.

28. Bisig, C.G.; Purro, S.A.; Contín, M.A.; Barra, H.S.; Arce, C.A. Incorporation of 3-nitrotyrosine into the $C$-terminus of alpha-tubulin is reversible and not detrimental to dividing cells. Eur. $J$. Biochem. 2002, 269, 5037-5045.

29. Sparaco, M.; Gaeta, L.M.; Tozzi, G.; Bertini, E.; Pastore, A.; Simonati, A.; Santorelli, F.M.; Piemonte, F. Protein glutathionylation in human central nervous system, potential role in redox regulation of neuronal defense against free radicals. J. Neurosci. Res. 2006, 83, 256-263.

30. Carletti, B.; Passarelli, C.; Sparaco, M.; Tozzi, G.; Pastore, A.; Bertini, E.; Piemonte, F. Effect of protein glutathionylation on neuronal cytoskeleton: A potential link to neurodegeneration. Neuroscience 2011, 192, 285-294.

31. Benitez-King, G.; Túnez, I.; Bellon, A.; Ortíz, G.G.; Antón-Tay, F. Melatonin prevents cytoskeletal alterations and oxidative stress caused by okadaic acid in N1E-115 cells. Exp. Neurol. 2003, 182, 151-159.

32. Yamanaka, Y.; Yoshida, S.; Doi, H. NGF-induced neurite outgrowth of PC12 cells in the presence of phosphatidylcholine hydroperoxides, implication for ageing. Mech. Aging Dev. 2008, $129,215-222$.

33. Liu-Snyder, P.; McNally, H.; Shi, R.; Borgens, R.B. Acrolein-mediated mechanisms of neuronal death. J. Neurosci. Res. 2006, 84, 209-218.

34. Gómez-Ramos, A.; Díaz-Nido, J.; Smith, M.A.; Perry, G.; Avila, J. Effect of the lipid peroxidation product acrolein on tau phosphorylation in neural cells. J. Neurosci. Res. 2003, 71, 863-870.

35. Counterman, A.E.; D’Onofrio, T.G.; Andrew, A.M.; Weiss, P.S. A physical model of axonal damage due to oxidative stress. Proc. Natl. Acad. Sci. USA 2006, 103, 5262-5266.

36. Yamashita, H.; Nakamura, K.; Arai, H.; Furumoto, H.; Fujimoto, M.; Kashiwagi, S.; Morimatsu, M. Electrophoretic studies on the phosphorylation of stathmin and mitogen-activated protein kinases in neuronal cell death induced by oxidized very-low-density lipoprotein with apolipoprotein E. Electrophoresis 2002, 23, 998-1004.

37. Neely, M.D.; Sidell, K.R.; Graham, D.G.; Montine, T.J. The lipid peroxidation product 4-hydroxynonenal inhibits neurite outgrowth, disrupts neuronal microtubules, and modifies cellular tubulin. J. Neurochem. 1999, 72, 2323-2333.

38. Mattson, M.P.; Fu, W.; Waeg, G.; Uchida, K. 4-hydroxynonenal, a product of lipid peroxidation, inhibits dephosphorylation of the microtubule-associated protein tau. Neuroreport 1997, 8, 2275-2281. 
39. Kaindl, A.M.; Sifringer, M.; Zabel, C.; Nebrich, G.; Wacker, M.A.; Felderhoff-Mueser, U.; Endesfelder, S.; von der Hagen, M.; Stefovska, V.; Klose, J.; et al. Acute and long-term proteome changes induced by oxidative stress in the developing brain. Cell Death Differ. 2006, 13, 1097-1109.

40. Rivas-Arancibia, S.; Guevara-Guzmán, R.; López-Vidal, Y.; Rodríguez-Martínez, E.; Zanardo-Gomes, M.; Angoa-Pérez, M.; Raisman-Vozari, R. Oxidative stress caused by ozone exposure induces loss of brain repair in the hippocampus of adult rats. Toxicol. Sci. 2010, 113, 187-197.

41. Hensley, K.; Christov, A.; Kamat, S.; Zhang, X.C.; Jackson, K.W.; Snow, S.; Post, J. Proteomic identification of binding partners for the brain metabolite lanthionine ketimine (LK) and documentation of LK effects on microglia and motoneuron cell cultures. J. Neurosci. 2010, 30, 2979-2988.

42. Chae, Y.C.; Lee, S.; Heo, K.; Ha, S.H.; Jung, Y.; Kim, J.H.; Ihara, Y.; Suh, P.G.; Ryu, S.H. Collapsin response mediator protein-2 regulates neurite formation by modulating tubulin GTPase activity. Cell Signal. 2009, 21, 1818-1826.

43. Fifre, A.; Sponne, I.; Koziel, V.; Kriem, B.; Yen Potin, F.T.; Bihain, B.E.; Olivier, J.L.; Oster, T.; Pillot, T. Microtubule-associated protein MAP1A, MAP1B, and MAP2 proteolysis during soluble amyloid beta-peptide-induced neuronal apoptosis. Synergistic involvement of calpain and caspase-3. J. Biol. Chem. 2006, 281, 229-240.

44. Santa-María, I.; Smith, M.A.; Perry, G.; Hernández, F.; Avila, J.; Moreno, F.J. Effect of quinines on microtubule polymerization, a link between oxidative stress and cytoskeletal alterations in Alzheimer's disease. Biochim Biophys. Acta 2005, 1740, 472-480.

45. Kabuta, T.; Setsuie, R.; Mitsui, T.; Kinugawa, A.; Sakurai, M.; Aoki, S.; Uchida, K.; Wada, K. Aberrant molecular properties shared by familial Parkinson's disease-associated mutant UCH-L1 and carbonyl-modified UCH-L1. Hum. Mol. Genet. 2008, 17, 1482-1496.

46. Boutte, A.M.; Woltjer, R.L.; Zimmerman, L.J.; Stamer, S.L.; Montine, K.S.; Manno, M.V.; Cimino, P.J.; Liebler, D.C.; Montine, T.J. Selectively increased oxidative modifications mapped to detergent-insoluble forms of Abeta and beta-III tubulin in Alzheimer's disease. FASEB J. 2006, 20, 1473-1483.

47. Reynolds, M.R.; Lukas, T.J.; Berry, R.W.; Biner, L.I. Peroxynitrite-mediated tau modification stabilize preformed filaments and destabilize microtubules through distinct mechanisms. Biochemistry 2006, 45, 4314-4326.

48. Tremblay, M.A.; Acker, C.M.; Davies, P. Tau phosphorylated at tyrosine 394 is found in Alzheimer's disease tangles and can be a product of the Abl-related kinase. J. Alzheimers Dis. 2010, 19, 721-733.

49. Minamide, L.S.; Striegl, A.M.; Boyle, J.A.; Meberg, P.J.; Bamburg, J.R. Neurodegenerative stimuli induce persistent $\mathrm{ADF} /$ cofilin-actin rods that dirupt distal neurite function. Nat. Cell Biol. 2000, 2, 628-636.

50. Whiteman, I.T.; Gervasio, O.L.; Cullen, K.M.; Guillemin, G.J.; Jeong, E.V.; Witting, P.K.; Antao, S.T.; Minamide, L.S.; Bamburg, J.R.; Goldsbury, C. Activated actin-depolymerizing factor/cofilin sequesters phosphorylated microtubule-associated protein during the assembly of alzheimer'like neuritic cytoskeletal striations. J. Neurosci. 2009, 29, 12994-13005. 
51. Santa-María, I.; Hernández, F.; Smith, M.A.; Perry, G.; Avila, J.; Moreno, F.J. Neurotoxic dopamine quinone facilitates the assembly of tau into fibrillar polymers. Mol. Cell. Biochem. 2005, 278, 203-212.

52. Balastik, M.; Lim, J.; Pastorino, L.; Lu, K.P. Pin1 in Alzheimer's disease, multiple substrates, one regulatory mechanism? Biochim. Biophys. Acta 2007, 1772, 422-429.

53. Zemlyak, I.; Sapolsky, R.; Gozes, I. NAP protects against Cytochrome C release, inhibition of the initiation of apoptosis. Eur. J. Pharmacol. 2009, 618, 9-14.

54. Divinski, I.; Holtser-Cochav, M.; Vulih-Schultzman, I.; Steingart, R.A.; Gozes, I. Peptide neuroprotection through specific interaction with brain tubulin. J. Neurochem. 2006, 98, 973-984.

55. Klein, R.L.; Dayton, R.D.; Henderson, K.M.; Petrucelli, L. Parkin is protective for substantia nigra dopamine neurons in a tau gene transfer neurodegeneration model. Neurosci. Lett. 2006, 401, 130-135.

56. Yang, Z.; Zhang, Q.; Ge, J.; Tan, Z. Protective effects of tetramethylpyrazine on rat retinal cell cultures. Neurochem. Int. 2008, 52, 1176-1187.

57. Ingber, D.E. From cellular mechanotransduction to biologically inspired engineering. Ann. Biomed. Eng. 2010, 38, 1148-1161.

58. Ingber, D.E. Tensegrity-based mechanosensing from macro to micro. Prog. Biophys. Mol. Biol. 2008, 97, 163-179.

59. Wang, N.; Naruse, K.; Stamenović, D.; Fredberg, J.J.; Mijailovich, S.M.; Tolić-Nørrelykke, I.M.; Polte, T.; Mannix, R.; Ingber, D.E. Mechanical behaviour in living cells consistent with the tensegrity model. Proc. Natl. Acad. Sci. USA 2001, 98, 7765-7770.

60. Lee, S.; Kolodziej, P.A. Short Stop provides an essential link between F-actin and microtubules during axon extension. Development 2002, 129, 1195-1204.

61. Subramanian, A.; Prokop, A.; Yamamoto, M.; Sugimura, K.; Uemura, T.; Betschinger, J.; Knoblich, J.A.; Volk, T. Shortstop recruits EB1/APC1 and promotes microtubule assembly at the muscle-tendon junction. Curr. Biol. 2003, 13, 1086-1095.

62. Houseweart, M.K.; Cleveland, D.W. Cytoskeletal linkers, new MAPs for old destinations. Curr. Biol. 1999, 9, R864-R866.

63. Dalpé, G.; Leclerc, N.; Vallée, A.; Messer, A.; Mathieu, M.; de Repentigny, Y.; Kothary, R. Dystonin is essential for maintaining neuronal cytoskeleton organization. Mol. Cell. Neurosci. 1998, 10, 243-257.

64. Yao, N.Y.; Broedersz, C.P.; Lin, Y.C.; Kasza, K.E.; Mackintosh, F.C.; Weitz, D.A. Elasticity in ionically cross-linked neurofilament networks. Biophys. J. 2010, 98, 2147-2153.

65. Kim, K.H.; Son, J.H. PINK1 gene knockdown leads to increased binding of parkin with actin filament. Neurosci. Lett. 2010, 468, 272-276.

66. Morot-Gaudry-Talarmain, Y. Physical and functional interactions of cyclophilin B with neuronal actin and peroxiredoxin-1 are modified by oxidative stress. Free Radic. Biol. Med. 2009, 47, 1715-1730.

67. Huang, T.Y.; Minamide, L.S.; Bamburg, J.R.; Bokoch, G.M. Chronophin mediates an ATP-sensing mechanism for cofilin dephosphorylation and neuronal cofilin-actin rod formation. Dev. Cell 2008, 15, 691-703. 
68. Barth, B.M.; Stewart-Smeets, S.; Kuhn, T.B. Proinflammatory cytokines provoke oxidative damage to actin in neuronal cells mediated by Rac1 and NADPH oxidase. Mol. Cell. Neurosci. 2009, 41, 274-285.

69. Oikawa, S.; Yamada, T.; Minohata, T.; Kobayashi, H.; Furukawa, A.; Tada-Oikawa, S.; Hiraku, Y.; Murata, M.; Kikuchi, M.; Yamashima, T. Proteomic identification of carbonylated proteins in the monkey hippocampus after ischemia-reperfusion. Free Radic. Biol. Med. 2009, 46, 1472-1477.

70. Loureiro, S.O.; Romão, L.; Alves, T.; Fonseca, A.; Heimfarth, L.; Neto, V.M.; Wyse, A.T.; Pessoa-Pureur, R. Homocysteine induces cytoskeletal remodelling and production of reactive oxygen species in cultured cortical astrocytes. Brain Res. 2010, 1355, 151-164.

71. Abrahan, C.E.; Insua, M.F.; Politi, L.E.; German, O.L.; Rotstein, N.P. Oxidative stress promotes proliferation and dedifferentiation of retina glial cells in vitro. J. Neurosci. Res. 2009, 87, 964-977.

72. Sasaki, T.; Gotow, T.; Shiozaki, M.; Sakaue, F.; Saito, T.; Julien, J.P.; Uchiyama, Y.; Hisanaga, S. Aggregate formation and phosphorylation of neurofilament-L Pro22 Charcot-Marie-Tooth disease mutants. Hum. Mol. Genet. 2006, 15, 943-952.

73. Rudrabhatla, P.; Zheng, Y.L.; Amin, N.D.; Kesavapany, S.; Albers, W.; Pant, H.C. Pin1-dependent prolyl isomerisation modulates the stress-induced phosphorylation of high molecular weight neurofilament protein. J. Biol. Chem. 2008, 283, 26737-26747.

74. Pierozan, P.; Zamoner, A.; Soska, A.K.; Silvestrin, R.B.; Loureiro, S.O.; Heimfarth, L.; Mello e Souza, T.; Wajner, M.; Pessoa-Pureur, R. Acute intrastriatal administration of quinolinic acid provokes hyperphosphorylation of cytoskeletal intermediate filament proteins in astrocytes and neurons of rats. Exp. Neurol. 2010, 224, 188-196.

75. Sparaco, M.; Gaeta, L.M.; Santorelli, F.M.; Passarelli, C.; Tozzi, G.; Bertini, E.; Simonati, A.; Scaravilli, F.; Taroni, F.; Duyckaerts, C.; Feleppa, M.; Piemonte, F. Friereich's ataxia, oxidative stress and cytoskeletal abnormalities. J. Neurol. Sci. 2009, 287, 111-118.

76. Shea, T.B.; Zheng, Y.L.; Ortiz, D.; Pant, H.C. Cyclin-dependent kinase 5 increases perikaryal neurofilament phosphorylation and inhibits neurofilament axonal transport in response to oxidative stress. J. Neurosci. Res. 2004, 76, 795-800.

77. Jeong, M.S.; Kang, J.H. Acrolein, the toxic endogenous aldehyde, induces neurofilament-L aggregation. BMB Rep. 2008, 41, 635-639.

78. Pamplona, R.; Dalfó, E.; Ayala, V.; Bellmunt, M.J.; Prat, J.; Ferrer, I.; Portero-Otín, M. Proteins in human brain cortex are modified by oxidation, glycoxidation, and lipoxidation. Effects of Alzheimer disease and identification of lipoxidation targets. J. Biol. Chem. 2005, 280, 21522-21530.

79. Ali, Z.S.; van Der Voorn, J.P.; Powers, J.M. A comparative morphologic analysis of adult onset leukodystrophy with neuronal spheroids and pigmented glia-A role for oxidative damage. J. Neuropathol. Exp. Neurol. 2007, 66, 660-672.

80. Wataya, T.; Nunomura, A.; Smith, M.A.; Siedlak, S.L.; Harris, P.L.; Shimohama, S.; Szweda, L.I.; Kaminski, M.A.; Avila, J.; Price, D.L.; et al. High molecular weight neurofilament proteins are physiological substrates of adduction by the lipid peroxidation product hydroxynonenal. J. Biol. Chem. 2002, 277, 4644-4648.

81. Sugimoto, K.; Yasujima, M.; Yagihashi, S. Role of advanced glycation end products in diabetic neuropathy. Curr. Pharm. Des. 2008, 14, 953-961. 
82. Chiasson, K.; Lahaie-Collins, V.; Bournival, J.; Delapierre, B.; Gélinas, S.; Martinoli, M.G. Oxidative stress and 17-alpha- and 17-beta-estradiol modulate neurofilaments differently. J. Mol. Neurosci. 2006, 30, 297-310.

83. Opii, W.O.; Joshi, G.; Head, E.; Milgram, N.W.; Muggenburg, B.A.; Klein, J.B.; Pierce, W.M.; Cotman, CW.; Butterfield, D.A. Proteomic identification of brain proteins in the canine model of human aging following a long-term treatment with antioxidants and a program of behavioural enrichment, relevance to Alzheimer's disease. Neurobiol. Aging 2008, 29, 51-70.

84. Mackenzie, G.G.; Salvador, G.A.; Romero, C.; Keen, C.L.; Oteiza, P.I. A deficit in zinc availability can cause alterations in tubulin thiol redox status in cultured neurons and in the developing fetal rat brain. Free Radic. Biol. Med. 2011, 51, 480-489.

85. Kanungo, J.; Zheng, Y.L.; Amin, N.D.; Pant, H.C. Targeting Cdk5 activity in neuronal degeneration and regeneration. Cell. Mol. Neurobiol. 2009, 29, 1073-1080.

(C) 2013 by the authors; licensee MDPI, Basel, Switzerland. This article is an open access article distributed under the terms and conditions of the Creative Commons Attribution license (http://creativecommons.org/licenses/by/3.0/). 